\title{
Tory II-C Performance as a Function of Flight Mach Number and Inlet Pressure Recovery
}

\author{
J. H. Moyer
}
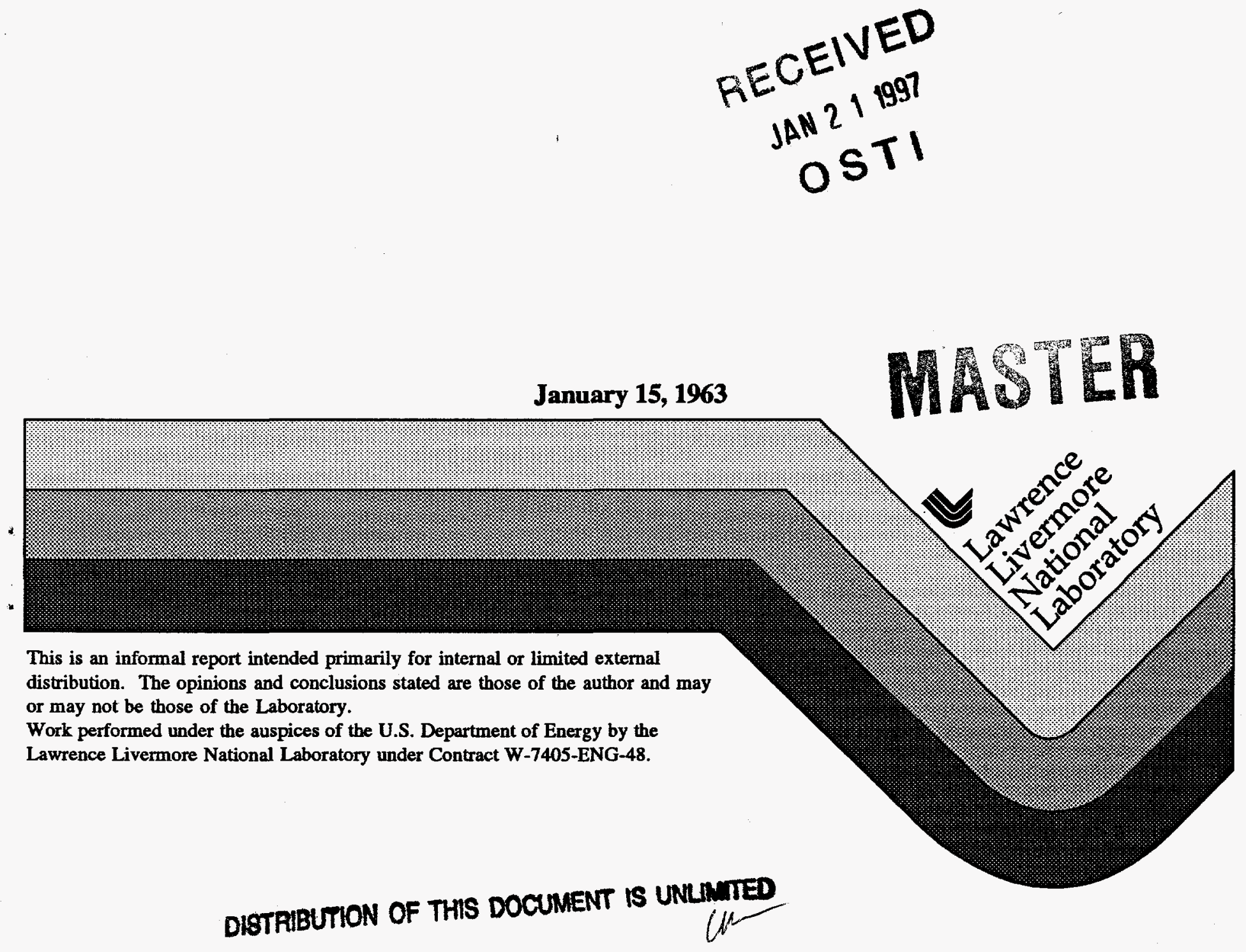


\section{DISCLAIMER}

This report was prepared as an account of work sponsored by an agency of the United States Government. Neither the United States Government nor any agency thereof, nor any of their employees, makes any warranty, express or implied, or assumes any legal liability or responsibility for the accuracy, completeness, or usefulness of any information, apparatus, product, or process disclosed, or represents that its use would not infringe privately owned rights. Reference herein to any specific commercial product, process, or service by trade name, trademark, manufacturer, or otherwise does not necessarily constitute or imply its endorsement, recommendation, or favoring by the United States Government or any agency thereof. The views and opinions of authors expressed herein do not necessarily state or reflect those of the United States Government or any agency thereof. 


\section{DISCLAMMER}

Portions of this document may be illegible in electronic image products. Images are produced from the best available original document. 


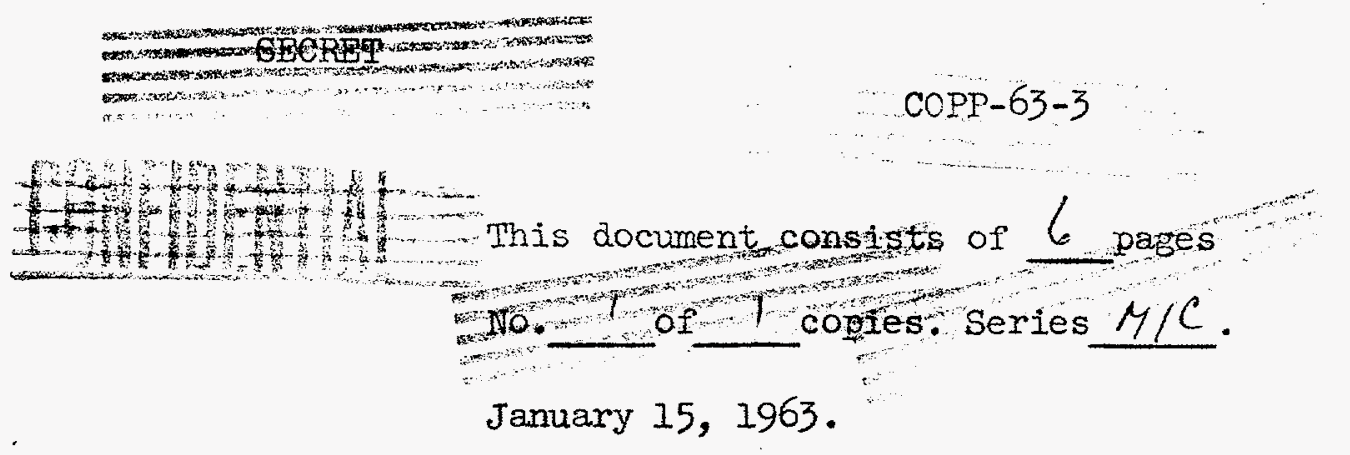

Naval Applications Memo No. 17. TO: Distribution DECHASIFICATION
STAMP ON REVERSE

FROM: $\quad$ J. H. Moyer

SUBJECT: Tory II-C Performance as a Function of Flight Mach Number and Inlet Pressure Recovery.

In a parameter study involving thrust vs. flight Mach number, M. D. Mintz ${ }^{1}$ has found that net jet thrust becomes a maximum at about Mach 3.66 if the Tory II-C design value of inlet pressure recovery is used at all Mach numbers. (Curve reproduced in Figure 1). This is not a realistic situation, however, because the attainable pressure recovery

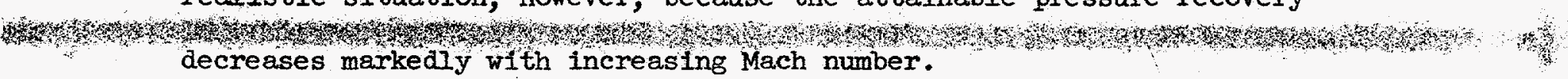
The effect of more realistic pressure recovery values may be seen in Figure 1. Two sets of values were employed, which differ by two percent, as shown in Table 1. Diffuser operation is always critical; i.e: we are using a "rubber" diffuser. Nozzle area has been optimized-for each operating condition.

Table 1 also lists some useful reactor parameters at or near the optimim nozzle area for each operating point. Figure 2 illustrates

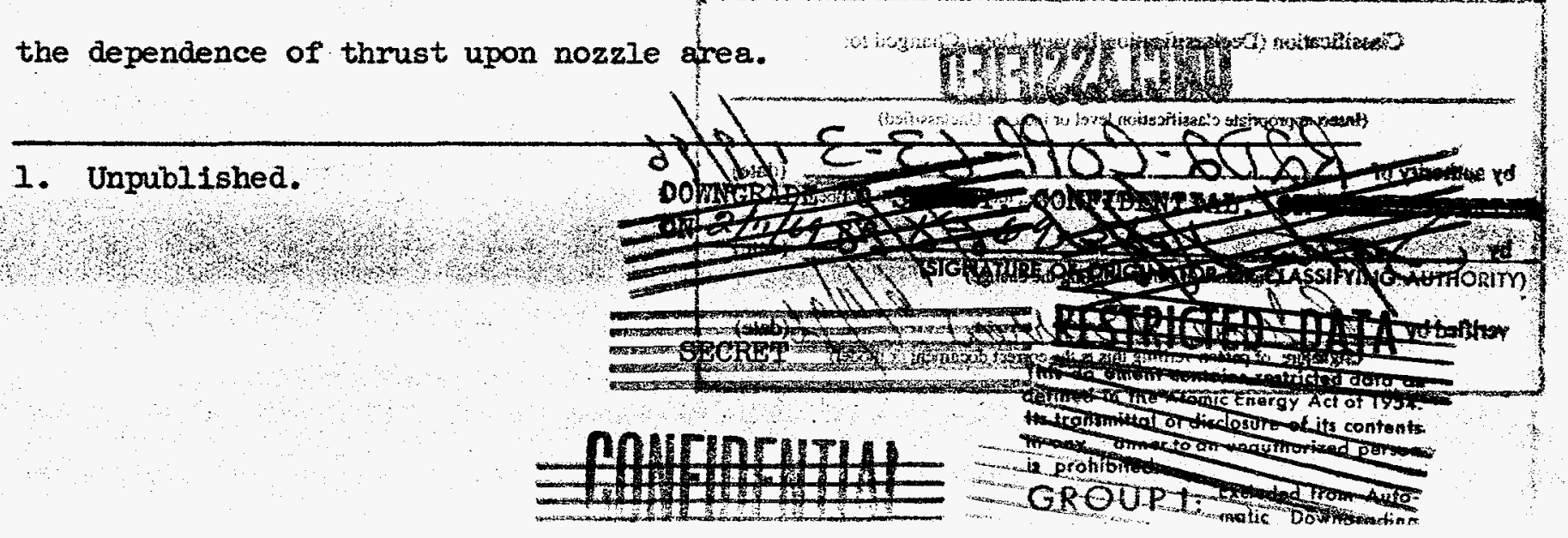



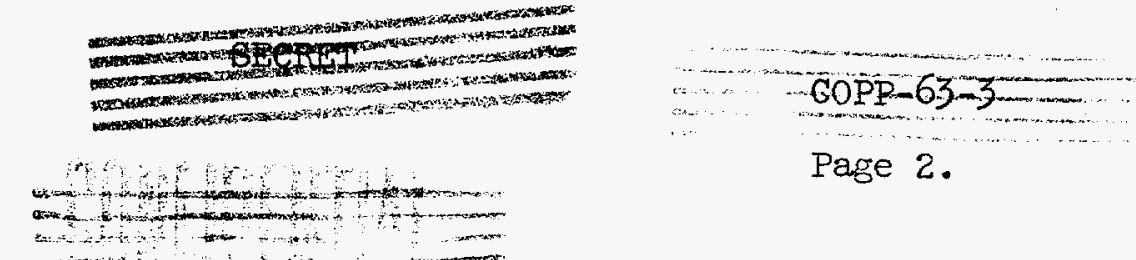

In Figure 3 is shewn-the variation of several reactor parameters

with nozzle area, for a flight Mach number of 3.0 and recovery of 0.76 .

A sirnilar graph for the Tory II-C design point may be found in Tory II-C

Memo 407, p. 75 .

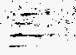

$J M: r p$

Distribution:

$1 / 36 \mathrm{~A}$

$2 / 36 \mathrm{~A}$

$3 / 36 \mathrm{~A}$

$4 / 36 A$

$5 / 36 \mathrm{~A}$

$6 / 36 A$

$7 / 36 \mathrm{~A}$

$8 / 36 A$

$9 / 36 \mathrm{~A}$

$10 / 36 \mathrm{~A}$

$11 / 36 \mathrm{~A}$

$12 / 36 \mathrm{~A}$

$13 / 36 \mathrm{~A}$

$14 / 36 \mathrm{~A}$

$1 5 \longdiv { 3 6 \mathrm { A } }$

$16 / 36 \mathrm{~A}$

$17 / 36 \mathrm{~A}$

$18 / 36 \mathrm{~A}$

$19 / 36 \mathrm{~A}$

$20 / 36 A$

$21 / 36 \mathrm{~A}$

$22 / 36 \mathrm{~A}$

$23 / 36 \mathrm{~A}$

$24 / 36 \mathrm{~A}$

$25 / 36 \mathrm{~A}$

$26 / 36 \mathrm{~A}$

$27 / 36 A$

$28 / 36 \mathrm{~A}$

$29 / 36 \mathrm{~A}$

$30 / 36 \mathrm{~A}$

$31 / 36 \mathrm{~A}$

$32 / 36 \mathrm{~A}$

$33 / 36 \mathrm{~A}$

$34 / 36 \mathrm{~A}$

$35 / 36 \mathrm{~A}$

$36 / 36 \mathrm{~A}$
A. Lorenz

$J$. Hadley

H. Reynolds

E. Goldberg

T. Stubbs

J. Moyer

M. Mintz

A. Cole

E. Sheridan

V. Hampel

C. Barnett

J. Thomas

M. Jester

H. Reynolds/File

H. Reynolds/The

H. Reynolds/File

H. Reynolds/File

H. Reynolds/File

H. Reynolds/File

W. B. Myers

W. Miller

W. Wells

E. Platt

C. Walter

$\mathrm{J}$. Hodges

J. Kane

A. Rothman

B. Rubin

H. McDonald

A. Kirschbaum

T. Merkle

P. Neal

J. G. Wenzel-Lockheed

J. Radcliffe, AEC-SAN

I. Hoffman, AEC-Weshington

H. Jaffee-AGI 


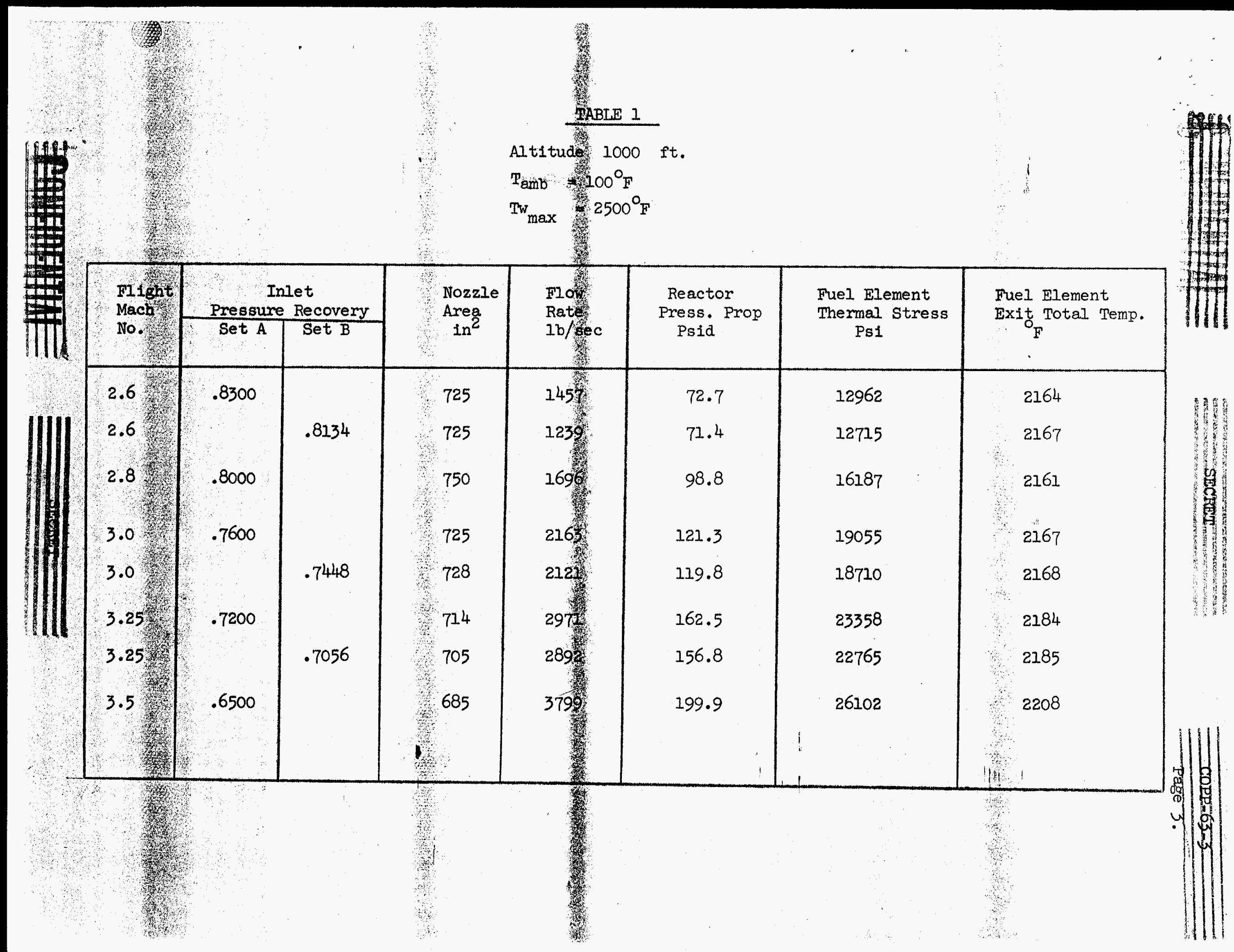



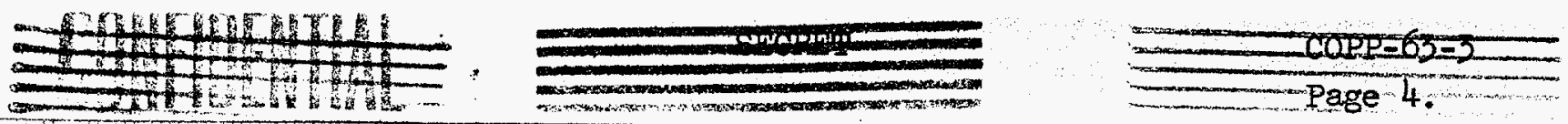

Ka

128
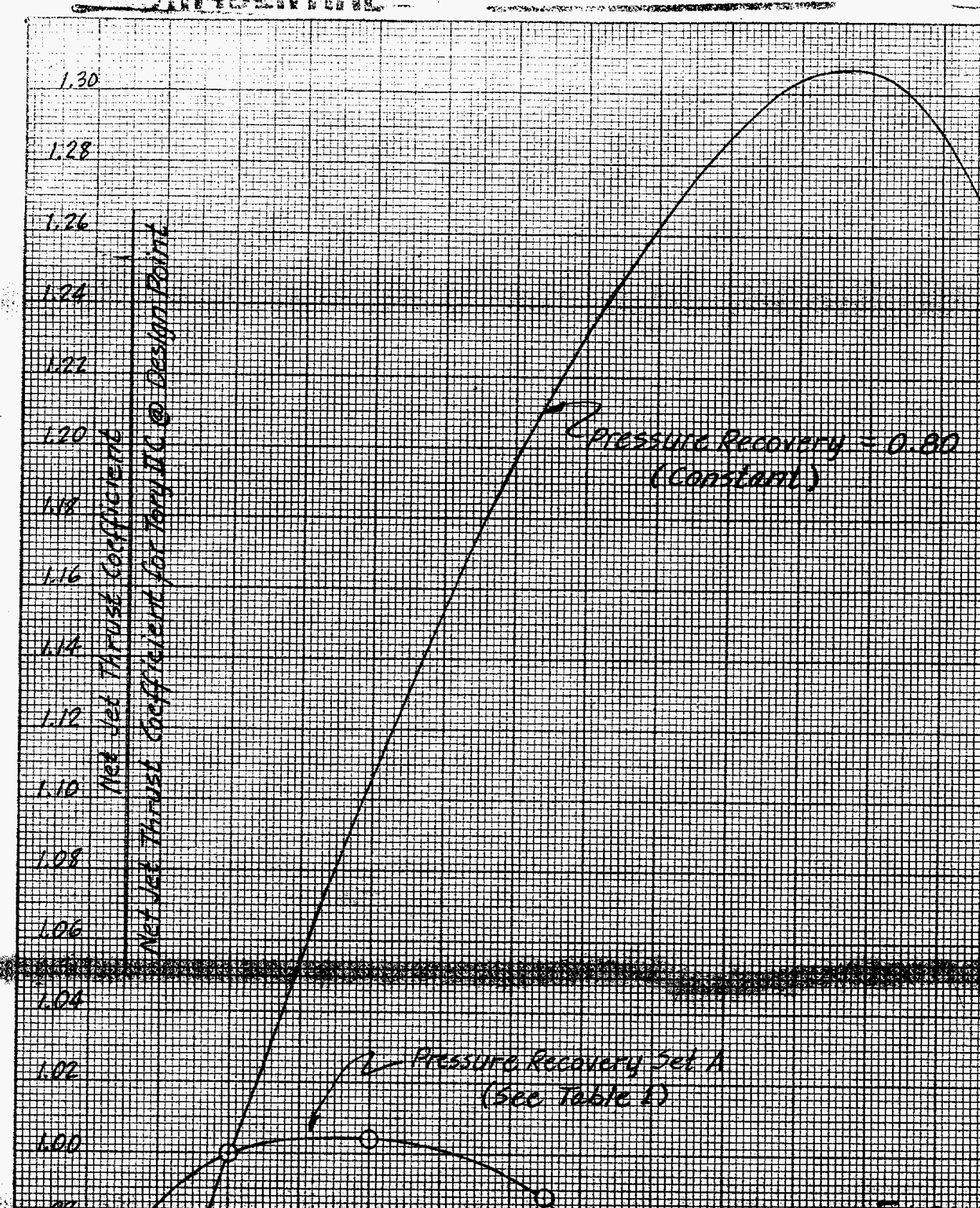

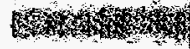

(Ł)

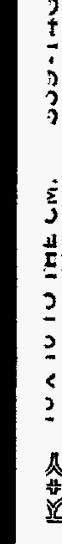

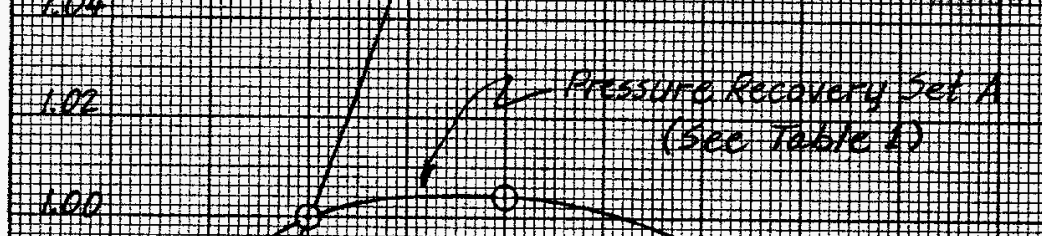

急

w

离

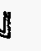

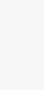

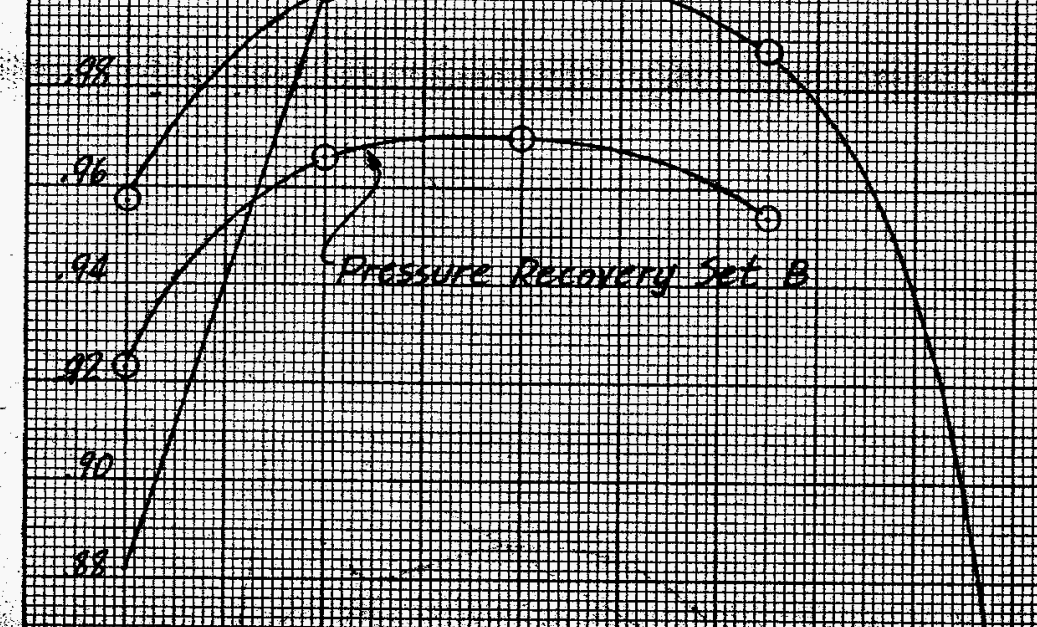

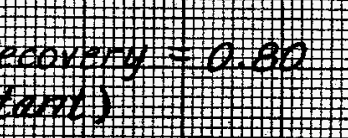




\section{1}

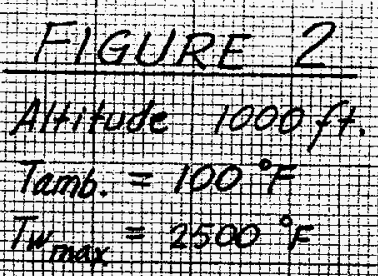

1.00

.98

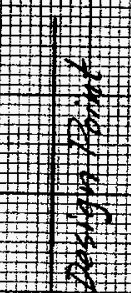

.97 +

$9 6 \longdiv { 1 }$

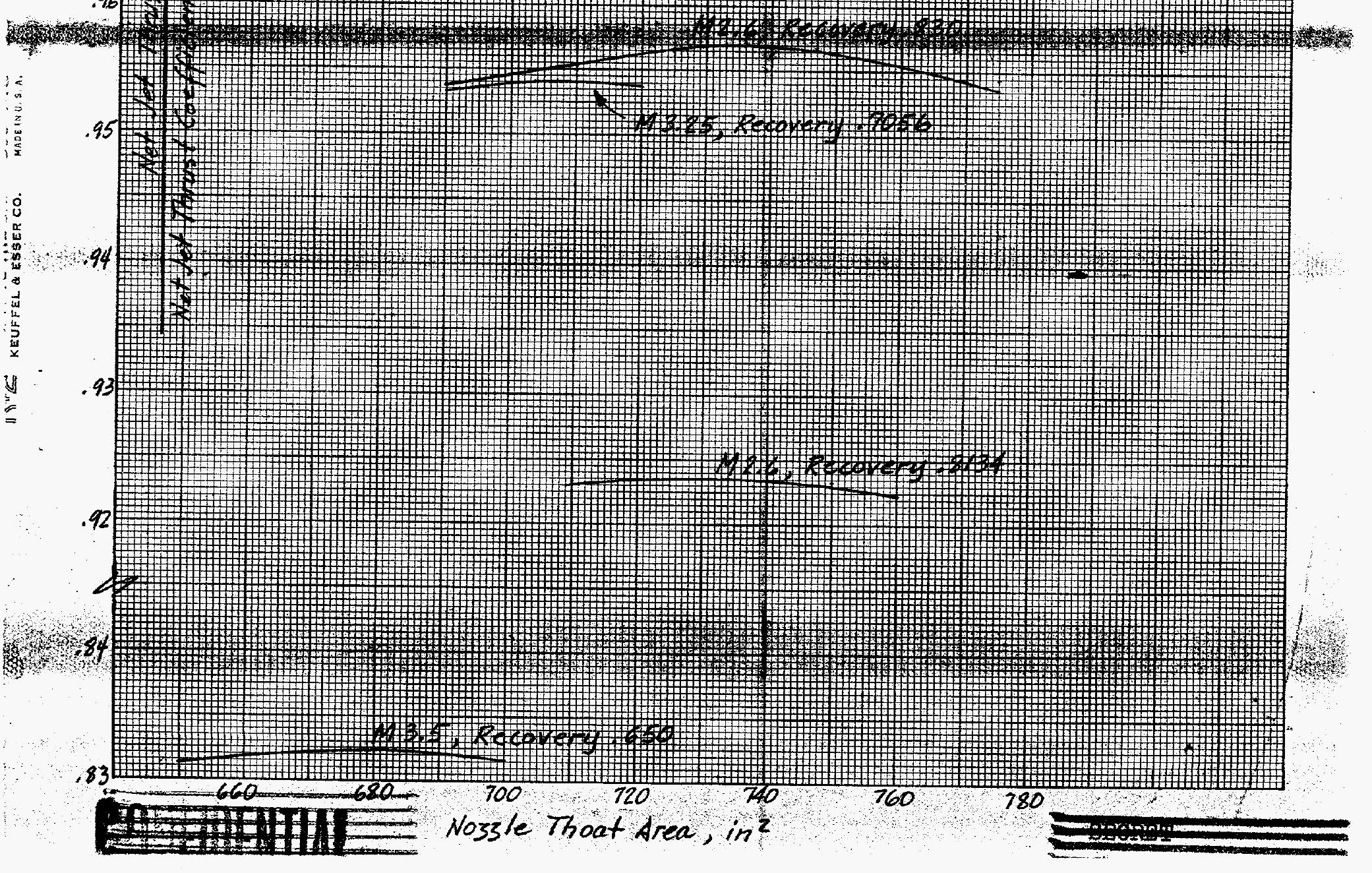




\section{$\frac{f 1 G U R E}{3}$ \\ $\operatorname{tamb}=100^{\circ} \mathrm{F}$ \\ Floh No. 30 \\ (2) 2500}

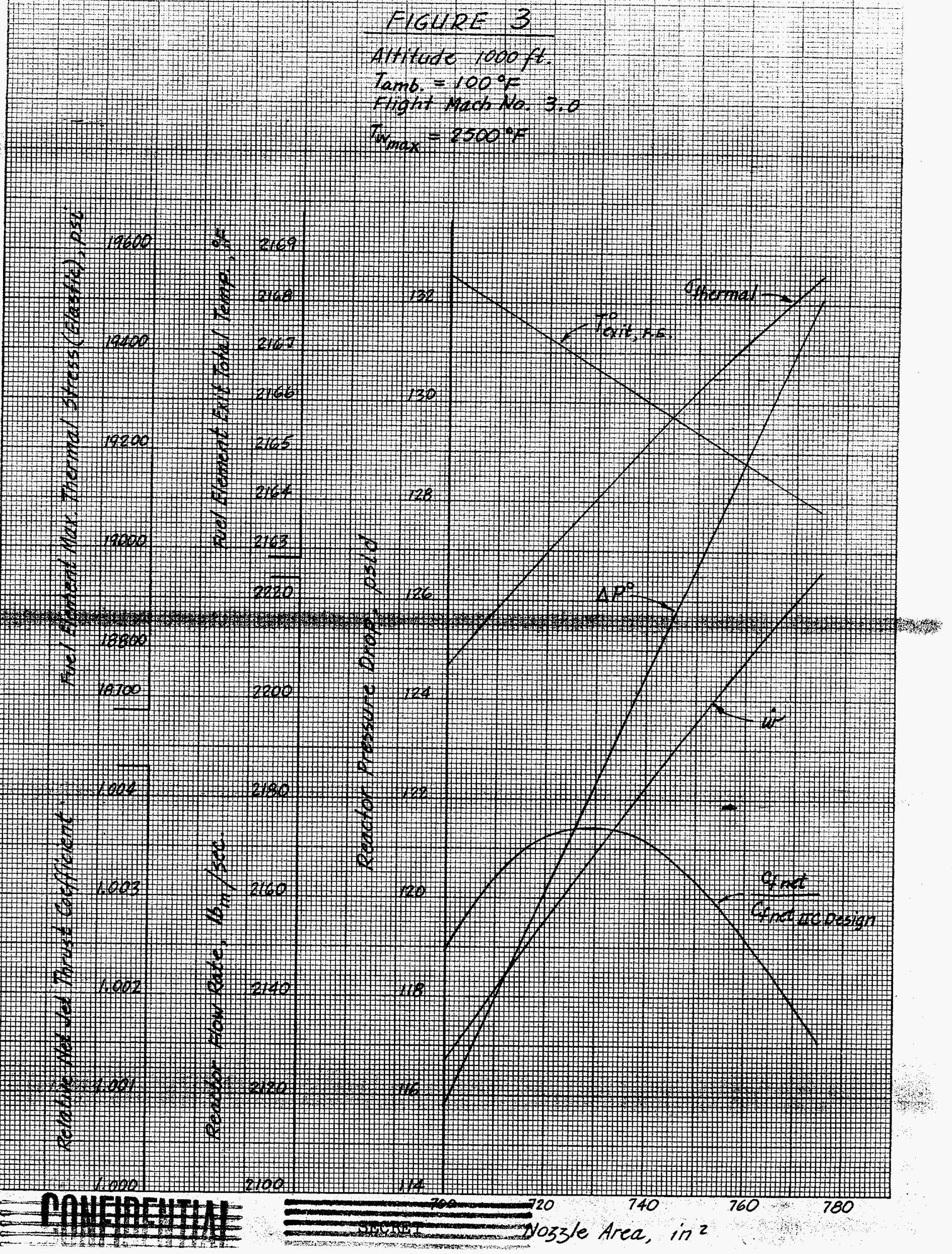

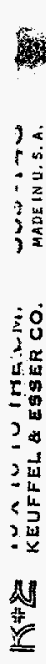
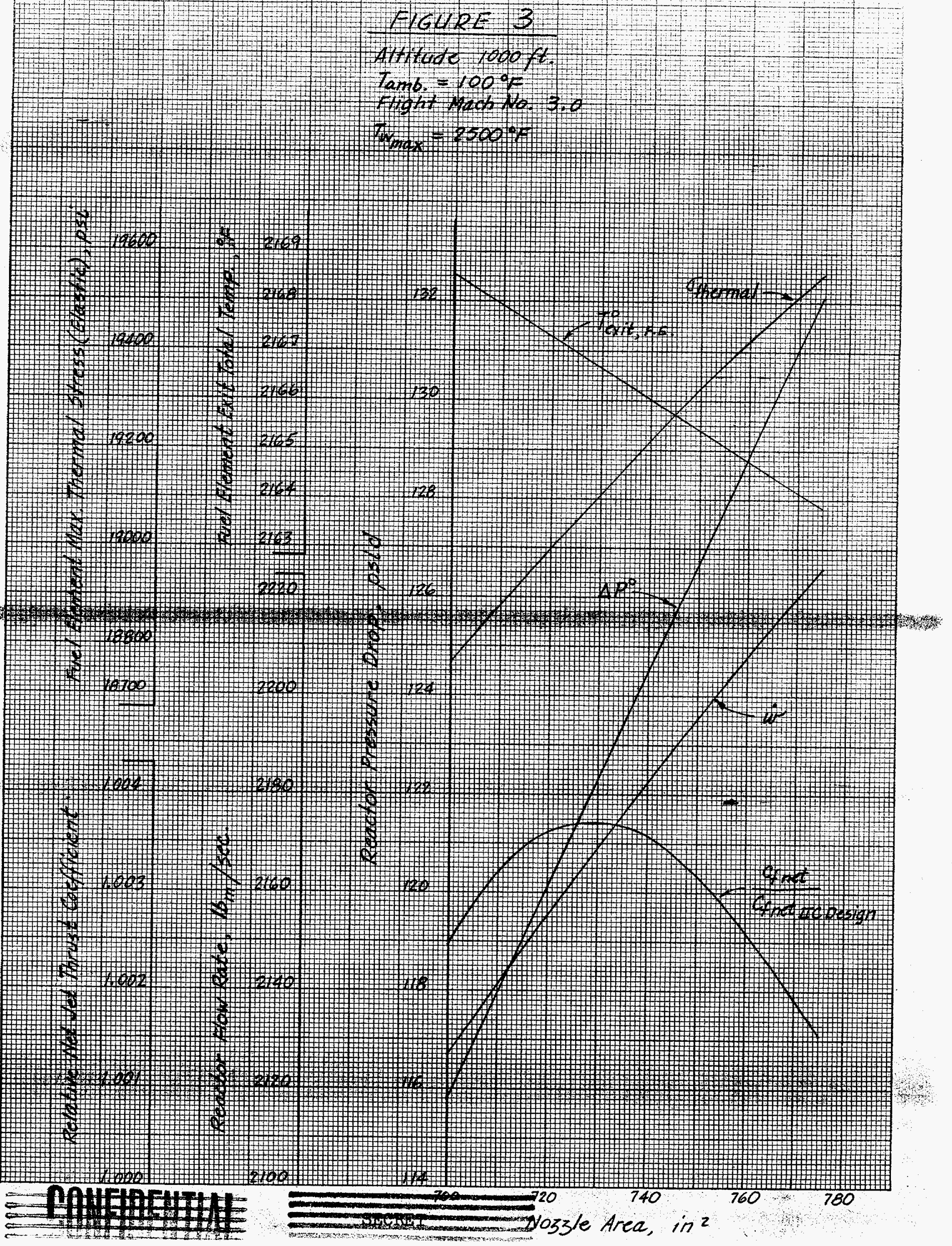